\title{
Fuzzy-based multi-kernel spherical support vector machine for effective handwritten character recognition
}

\author{
A K SAMPATH* and N GOMATHI \\ Veltech Dr.R.R \& Dr.S.R. Technical University, Avadi, Chennai 600 062, India \\ e-mail: sampath.ak127@gmail.com
}

MS received 17 August 2016; revised 18 December 2016; accepted 18 January 2017; published 8 August 2017

\begin{abstract}
Due to constant advancement of computer tools, automated conversion of images of typed, handwritten and printed text is important for various applications, which has led to intense research for several years in the field of offline handwritten character recognition. Handwritten character recognition is complex because characters differ by writing style, shapes and writing devices. To resolve this problem, we propose a fuzzy-based multi-kernel spherical support vector machine. Initially, the input image is fed into the pre-processing step to acquire suitable images. Then, histogram of oriented gradient (HOG) descriptor is utilised for feature extraction. The HOG descriptor constitutes a histogram estimation and normalisation computation. The features are then classified using the proposed classifier for character recognition. In the proposed classifier, we design a new multi-kernel function based on the fuzzy triangular membership function. Finally, a newly developed multi-kernel function is incorporated into the spherical support vector machine to enhance the performance significantly. The experimental results are evaluated and performance is analysed by metrics such as false acceptance rate, false rejection rate and accuracy, which is implemented in MATLAB. Then, the performance is compared with existing systems based on the percentage of training data samples. Thus, the outcome of our proposed system attains $99 \%$ higher accuracy, which ensures efficient recognition performance.
\end{abstract}

Keywords. Handwritten characters; HOG descriptor; kernel function; fuzzy triangular membership function; support vector machine (SVM).

\section{Introduction}

Handwriting recognition has become one of the fascinating techniques in some applications such as image processing, pattern recognition, artificial intelligence and machine learning. This technique can exploit intelligible handwritten input from different sources such as photographs, touch screens, paper documents and other devices [1]. The handwritten image is sensed in the computer by optical scanning method (optical character recognition). Optical character recognition (OCR) is a process that converts the printed or handwritten file into its corresponding text format. The OCR - into two types: offline and on-line character recognition [2]. Further, the offline character recognition is split into two aspects: handwritten and machine printed character. An offline character is recognised by writing the characters using pen and then translating into optically scanned images. Nowadays, offline character is used in wide variety of application such as official document reading, postal sorting and reading bank cheque. The characters are written and apprehended by some electronic

*For correspondence hardware such as a smart pen and pressure-sensitive tablets, which is used to determine the pressure and velocity of the smart pen [3].

In OCR, three significant steps are prerequisite for recognising a character image: (i) preprocessing; (ii) feature extraction; and (iii) classification. The input images are preprocessed to obtain the image concisely for subsequent steps. The preprocessing methods include resizing, normalisation, and extraction of region of interest (ROI) extraction [4]. Then, feature extraction is an important aspect in OCR system since it provides the features as input to the classifier to recognise the image. Several feature extraction methods are projection histograms, template matching, zoning, contour profiles, gradient descriptors, spline curve approximations and Gabor features [5]. Feature extraction by each character addresses the problems of different writing style, size and shape variation. Gradient feature extraction is a new emerging technique in handwritten character recognition. The gradient feature extracts the local, texture and edges features. The gradient features are acquired by the horizontal and vertical gradients. The main objective of the gradient feature is that the features are extracted from grayscale as well as binary 
images and also the performance against contour noise is enhanced [6].

Efficient classifier is utilised in the classification stage to enhance the character recognition performance. Classifiers such as Neural Network, Nearest Neighbour classifier, Multilayer perceptron, Support Vector Machine classifier, and Hidden Markov Model [7] are employed. While classifying the features, there are two constraints which mitigate the recognition performance: (i) the character may not be correlated with the training data samples since the character is varied in style and size; and (ii) the handwritten characters are vague, and some features contain errors. To resolve these problems, we can classify the feature by both dynamic and static representation [8]. Nowadays, the SVM classifier is preferred for the recognition system because it requires less computation time than other classifiers. The classification algorithm can be categorised into two types: discriminative approach and model-based approach. The discriminative approach is utilised to provide better separation of features among all the classes in the classifier but cannot compact with the outliers. There is possibility of outlier detection in model-based approach [2, 9]. Thus, handwritten characters are recognised by an efficient classifier which ensures better-accuracy performance.

In this paper, fuzzy-based spherical SVM classifier is proposed for handwritten character recognition. The main objective is to attain higher-accuracy recognition performance. To achieve this objective, the input character image is recognised using the feature extraction and classification. Initially, an input image is preprocessed by the resizing operation. Thus, the preprocessed image is obtained concisely, which is used to extract the features and classification. Then, the resultant image is given as input to extract the character features. The HOG descriptor is employed here to extract the features by estimating the histogram and normalising of the image. Finally, we proposed a novel fuzzy-based spherical SVM classifier for recognition performance to enhance accuracy. In the novel classifier, first, we develop a new kernel function by tangential and exponential kernel. Subsequently, the weights in kernel function are evaluated by fuzzy triangular membership function. Secondly, the newly developed kernel function is embedded into the spherical SVM classifier. Thus, the proposed fuzzy-based multi-kernel spherical SVM classifier attains better character recognition performance.

The main contributions of this paper are as follows:

- We design a new kernel function that is composed of both exponential and tangential kernels based on the triangular fuzzy membership function.

- A novel fuzzy-based spherical SVM classifier is proposed which exploits the newly designed kernel function to acquire the high-accuracy performance.

This paper is structured as follows. Section 2 discusses the handwritten character recognition systems from eight research papers. Section 3 explains the problem statement and challenges behind the OCR. Section 4 describes the proposed methodology by the HOG descriptor and fuzzybased multi-kernel spherical SVM classifier. Section 5 demonstrates the experimental results and comparative performance analysis. Section 6 presents the conclusions.

\section{Literature review}

Kamble and Hegadi [1] presented the rectangle histogram oriented gradient representation as the basis for extraction of features. These algorithms required a few simple arithmetic operations per image pixel, which made them suitable for real-time applications. Our dataset consists of 8,000 samples each of 40 basic handwritten Marathi characters. Among these, 10 samples of each character from different writers were collected. All sample images of handwritten Marathi characters were normalised to $20 \times 20$ pixel size. The description of the algorithm and experiment with our data set was presented in this paper. Experimental results using support vector machines (SVMs) and feedforward artificial neural network (FFANN) classification techniques were evaluated. Our results demonstrated that the high performance of these features when classified using FFANN classification.

Basu et al [10] proposed a hierarchical approach for OCR of handwritten Bangla words. The presented approach was used to segment a word image on Matra hierarchy, then recognised the individual word segments and finally identified the constituent characters of the word image through an intelligent combination of recognition decisions of the associated word segments. Due to possible appearances of consecutive characters of Bangla words on overlapping character positions, segmentation of Bangla word images was not easy. For successful OCR of handwritten Bangla text, not only recognition but also segmentation of word images was important. In this aspect, the presented approach was dealt with both segmentation and recognition of handwritten Bangla word images for a complete solution to handwritten word recognition problem, an essential area of OCR of handwritten Bangla text. In dealing with a certain category of word segments, created on Matra hierarchy, a sophisticated recognition technique, namely, twopass approach. The degree of sophistication of the classification technique was also rationally tuned and the recognition of middle-zone-modified shapes of Bangla script was done through simple template matching. Considering learning and generalisation abilities of multi-layer perceptrons (MLPs), MLP-based pattern classifiers were used here for most of the classification-related tasks. A powerful feature set was also designed under this work for recognition of complex character patterns using three types of topological features, namely, longest run, modified shadow and octant centroid features. Briefly, the work was 
dealt with a practical problem of OCR of Bangla text involving recognition as well as segmentation of constituent characters of handwritten Bangla words.

Bhowmik et al [2] explained the SVM-based hierarchical classification schemes for recognition of handwritten Bangla characters. A comparative study was made on MLP, radial basis function network and SVM classifier for 45 class recognition problems. SVM classifier was found to outperform the other classifiers. A fusion scheme using the three classifiers was proposed, which was marginally better than the SVM classifier. It was observed that the groups of all the characters had the similar shapes. These groups were determined in two different ways on the basis of the confusion matrix obtained from SVM classifier. In the former, the groups were disjoint while they were overlapped in the latter. Another grouping scheme was proposed based on the confusion matrix obtained from the neural gas algorithm. Groups were disjoint here. Three different two-stage hierarchical learning architectures (HLAs) were proposed using the three grouping schemes. An unknown character image was classified into a group in the first stage. The second stage was to recognise the class within this group. Performances of the HLA schemes were found to be better than single-stage classification schemes.

Katiyar and Mehfuz [3] presented the combined multiple features extracted using seven different approaches. This approach was utilised to achieve better accuracy and reduced computational time for recognition of handwritten characters using Genetic Algorithm which optimised the number of features along with a simple and adaptive MLP classifier. Experiments were performed using a standard database of Centre of Excellence for Document Analysis and Recognition (CEDAR) for English alphabet. Finally, the experimental results were obtained from this database, which ensured the effectiveness of this system.

Cheng-Lin Liu [6] described the gradient direction histogram feature for the handwritten character recognition performance. To alleviate the effect of stroke direction distortion caused by shape normalisation and provided the higher recognition accuracies, we proposed a feature extraction approach, called normalisation co-operated gradient feature (NCGF) extraction, which was utilised to map the gradient direction elements of original image to direction planes without generating the normalised image and could be combined with various normalisation methods. Experiments on handwritten Japanese and Chinese character databases were made and then compared with the normalisation-based gradient feature. Thus, the NCGF approach was reduced the recognition error rate by factors ranging from $8.63 \%$ to $14.97 \%$ with high confidence of significance when combined with pseudo two-dimensional normalisation.

Pradeep et al [5] proposed an offline handwritten English character recognition system using hybrid feature extraction technique and neural network classifiers. A hybrid feature extraction method is composed of both the diagonal and directional-based features. The proposed system suitably combined the salient features of the handwritten characters to enhance the recognition accuracy. Neural Network (NN) topologies, namely, back propagation neural network and radial basis function network, were built to classify the characters. The k-nearest neighbour network was also built for comparison. The feed-forward NN topology exhibited the highest recognition accuracy and was identified to be the most suitable classifier. The proposed system was helpful for postal or parcel address recognition and conversion of any handwritten document into structural text form. The experimental results were calculated and then the performance of the recognition systems was compared extensively using test data with existing systems.

De Stefano et al [11] illustrated the Genetic Algorithm (GA)-based feature selection algorithm in which feature subsets were evaluated using a specifically devised separability index. This index was employed to measure the statistical properties of the feature subset and independent of any specific classification scheme. The proposed index represented the extension of the Fisher Linear Discriminant method and used the covariance matrices for estimating how class probability distributions were spread out in the considered $\mathrm{N}$-dimensional feature space. Our approach did not require any a priori knowledge about the number of features to be used in the feature subset. Experiments had been performed using three standard databases of handwritten digits and a standard database of handwritten letters, while the solutions were found which had been tested with different classification methods. Then, the results were compared with those results obtained using the whole feature set and with those obtained using standard feature selection algorithms. The comparison performance of GAbased feature selection confirmed the effectiveness of our approach.

Surinta et al [12] explained the local gradient feature descriptors, namely, the scale invariant feature transform keypoint descriptor and the HOGs for handwritten character recognition. Local gradient feature descriptors were used to extract the feature vectors from the handwritten images, which were then presented to a machine learning algorithm to do the actual classification. As classifiers, the k-nearest neighbour and the SVM algorithms were used. We evaluated these feature descriptors, and the classifiers were utilised for recognising the three different language scripts, namely Thai, Bangla and Latin, consisting of both handwritten characters and digits. The results proved that the local gradient feature descriptors significantly outperformed using pixel intensities from the images. When the proposed feature descriptors were combined with the SVM, very high accuracies were obtained on the Thai handwritten datasets (character and digit), the Latin handwritten datasets (character and digit) and the Bangla handwritten digit dataset. 


\section{Motivation}

\subsection{Problem statement}

Handwritten character recognition is the process of conversion of handwritten text into machine-readable form. The major problem is to recognise the characters from documents, photos because of a distinct way of writing the characters. Let $I$ be the input image that consists of $h$ number of the handwritten character image. It is expressed by

$$
I=I_{l} ; \quad 1 \leq l \leq h
$$

where $I$ is the input image of our proposed system and $h$ is the number of the character image. Here, the main challenge is to recognise the character precisely using the proposed classifier.

\subsection{Challenges}

- The OCR becomes one of the challenging techniques for several applications such as the text entry in the computer is automatically done for the desktop application, library cataloging and ledgering, language processing, aid for the blind to read the documents [13].

- Recognition of handwritten character [14] about any language is a major challenge since each character varies from person to person and also differs by their way of style.

- The extraction of feature space from the image is another challenge as the inconsistency is set up by the different writing style, rotation and deformation [15].

- Due to the shape variation in handwritten characters, using a competent classifier is a challenge one faces when enhancing the recognition performance because the shape variation causes the misclassification [16].

\section{Proposed methodology: handwritten character recognition using fuzzy-based multi-kernel spherical SVM classifier}

The aim of this paper is to recognise the character image efficiently using HOG descriptors and fuzzy-based spherical SVM classifier. The proposed work considers the number of handwritten characters for recognition. Figure 1 shows the block diagram representation of proposed methodology. The proposed methodology comprises three following steps: preprocessing, feature extraction and classification. As a first step, the input character image is fed into the preprocessing step, which obtains the image concisely for further steps. Subsequently, the preprocessed image is given as input to the HOG descriptor. Here, the

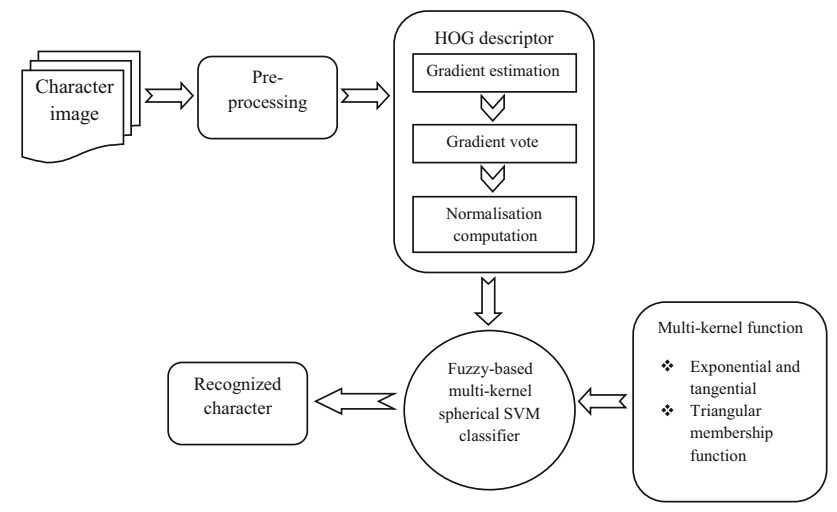

Figure 1. Block diagram representation of proposed methodology.

HOG descriptor is utilised for feature extraction. The HOG descriptor constitutes gradient estimation, gradient vote and normalisation computation. Thus, the character features are extracted from each block of the image. Finally, the features are classified by the proposed fuzzy-based spherical SVM classifier. In the proposed classifier, a novel kernel function is designed using two different kernel functions based on the fuzzy triangular membership function. This new kernel function is then employed in the spherical SVM classifier to ensure better recognition performance.

\subsection{Preprocessing}

Preprocessing is the initial step for the image recognition system because it improves the reliability of the image. In character recognition system, the preprocessing technique is utilised to acquire an image suitable for the subsequent steps. It also tends to mitigate or intensify certain image details for faster evaluation. Here, the resizing operation is utilised to attain the preprocessed character image $I$ concisely. The preprocessing stage is used for noise removal, image enhancement and character normalisation. Figure 2 shows the sample character image of our proposed system.

\subsection{Feature extraction using $H O G$}

The preprocessed image is then fed into the extraction of the feature. The HOG descriptor [17] is utilised for feature computation. The advantage of using HOG over other descriptors is it emulates and encodes the gradients in each character, and is vigorous to invariance in the local geometric and photometric transformations and illumination variation. This descriptor counts the occurrences of gradient orientation in the localised portion of an image. The main purpose behind this descriptor is to distinguish the local object appearance and shape within an image assist with the knowledge of intensity gradients or edge direction. Here, we consider two computation units: cells and blocks. 


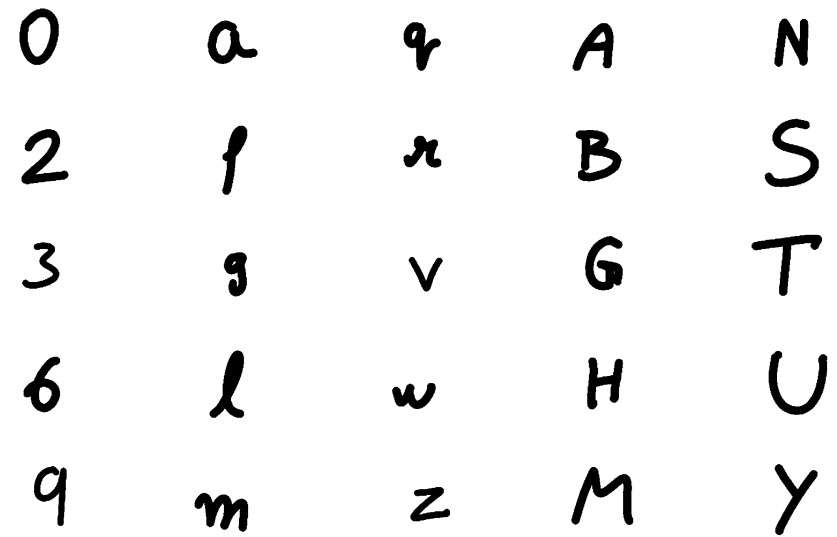

Figure 2. Sample input images.

Initially, the HOG feature descriptor is exploited by dividing the image into small regions called cells. In each cell, the histogram of gradient directions or edge orientations is computed for the pixels. Then, to improve accuracy, all the histograms in each cell are normalised. The normalisation is evaluated by the measure of the intensity of a group of cells called blocks. Figure 3 represents the schematic representation of HOG feature extraction.

The preprocessed image is divided into $g$ number of cells as shown in figure 3. Each cell has the size $X \times Y$, which contains $X * Y$ number of pixels. The histogram is then estimated in each cell. Then, the $r$ number of blocks is generated by grouping of cells. Thus, the normalisation is computed in each block of an image. In figure 3 , if each cell in the image has $4 \times 4$ size, then each block is represented by $16 \times 16$ size of the image. Thus, the HOG feature extraction is elucidated by the three following steps:

(i) Gradient estimation: For each pixel $(x, y)$ in the cell, the magnitude and orientation are computed. Let the pixel be located at coordinate $(x, y)$ and its luminance value denoted by $l(x, y)$. Then, the horizontal and vertical gradients of the pixels are represented by

$$
\begin{aligned}
& I_{x}(x, y)=l(x+1, y)-l(x-1, y) \\
& I_{y}(x, y)=l(x, y+1)-l(x, y-1) .
\end{aligned}
$$

Then, the [17] magnitude $m(x, y)$ is defined as

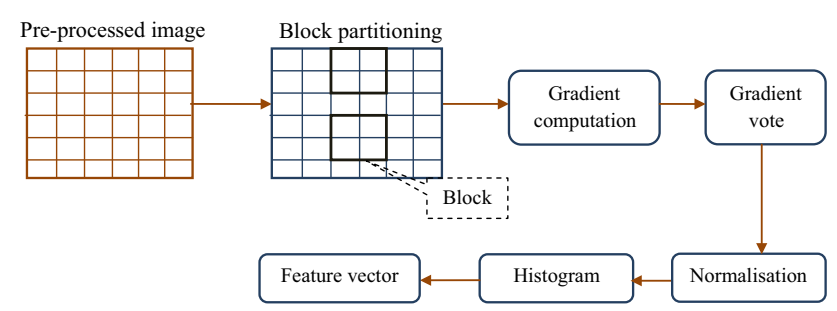

Figure 3. Schematic representation of HOG feature extraction.

$$
m(x, y)=\sqrt{I_{x}(x, y)^{2}+I_{y}(x, y)^{2}} .
$$

Then, the direction is computed by

$$
\theta(x, y)=\arctan \frac{I_{x}(x, y)}{I_{y}(x, y)} .
$$

(ii) Gradient vote: After the magnitude and orientation of each pixel is calculated, it leads to evaluation of a gradient vote for an orientation histogram with respect to the gradient element centred within the cell. Then, the orientation is determined, which is divided into a number of bins. The two bins are $1-a$ and $a$, which is incremented by the following equation:

$$
\begin{gathered}
v_{1}=(1-a) * m(x, y) \\
v_{2}=a * m(x, y)
\end{gathered}
$$

where $a$ is the weight of the pixel and $m(x, y)$ is the magnitude. Then, the weight of each pixel is calculated as

$$
a=(c+0.5)-\frac{b * \theta(x, y)}{\pi}
$$

where $c$ is the bin in which $\theta(x, y)$ belongs and $b$ is the total number of bins. Finally, the normalisation is evaluated in each block.

(iii) Normalisation computation: The normalisation is computed in each block, which is composed of a number of histogram cells as shown in figure 3 . Thus, the normalisation for each block is represented as

$$
n_{u}^{c}=\frac{n_{u}}{\sqrt{\|n\|_{2}^{2}+\sigma^{2}}}
$$

where $n_{u}$ is the vector of the block which belongs to the combined histogram cells and $u$ is a number which ranges from one to $g \times b$ and $\sigma$ is defined as a small constant to restrict the infinite constraint. Thus, the feature vector $F$ is formed by collecting the HOG descriptors from all the blocks in the image.

\subsection{Fuzzy-based spherical SVM classifier for recognition}

After the features are extracted using HOG descriptors, then it is fed into the classifier to recognise the character image. In general, the SVM classifier is designed earlier for two classifications and used to resolve the problem when the feature constitutes small samples, high-dimensional data, non-linearity, etc. However, the SVM contains low propensity to grip the large datasets of the feature, which leads to degrading the accuracy in performance. Thus, the fuzzy-based multi-kernel spherical SVM classifier is utilised in this paper. The kernel function is newly developed based on the fuzzy membership function, which is 
integrated into the spherical SVM classifier [18] to attain better-accuracy performance. The design of kernel function is a significant aspect for the classifier, which implicitly identifies the high-dimensional feature space data where a maximal margin hyperplane is found. Then, the kernel function contains $\alpha$ and $\beta$-the two parameters which are computed by the fuzzy triangular membership function. The membership function in the fuzzy logic represents the degree of truth as an extension of valuation. The membership function is also to provide the indicator functions in classical sets. Thus, the fuzzy-based spherical SVM classifier exploits different kernel function which exhibits better character recognition performance.

4.3a Design a new kernel function using the triangular membership function: The kernel method has been widely used in the SVM and that depends on the data which is expressed only through the dot products between the image arguments. The idea behind the kernel function is to grant a bridge from the linear data to the non-linear separable data. The kernel function consists of two advantages: (i) it has the tendency to create non-linear decision boundaries for the classifier and (ii) the kernel function is employed to let the user apply the data into the classifier without fixeddimensional vector space representation. Here, the two kernel functions such as exponential and tangential are utilised which simulate the higher-dimensional vector where the classifier efficiently recognises the character image. Thus, the newly developed kernel is expressed by

$$
K=\alpha \exp \left(-\left(V_{c} * V_{d}\right)\right)+\beta \tanh \left(-\left(V_{c} \cdot * V_{d}\right)\right)
$$

where $\alpha$ and $\beta$ are the weights which are computed by the fuzzy triangular membership function. The exponential kernel function is defined as same as the Gaussian kernel function without using the square norm of the function. It also belongs to the radial-basis kernel function. The tangential kernel is also known as the sigmoid or multilayer perceptron kernel function. This kernel function is quite popular for SVM classifier due to origin from the NN theory. Thus, the weights are estimated using the triangular membership function.

Triangular membership function: The fuzzy membership function demonstrates that each point in the input space is mapped to a membership value (or degree of membership). Thus, the degree of the membership function is conceptually discrete since the fuzzy truth defines the membership in vaguely defined sets. The fuzzy set contains different membership functions such as triangular, trapezoidal, and Gaussian. In this paper, we utilise the triangular membership function for new kernel function. The triangular membership function is defined by three limits, which are represented as lower value, middle value and upper value. The triangular membership function has less computation time and better efficiency when compared to other membership function. Thus, the membership function is expressed as follows:

$$
z=V_{c} * V_{d}
$$

where $z$ is the value which is determined by the product of two violating vectors. The triangular membership function is used here to compute $\alpha$ value. The triangular membership function is represented by

$$
t(\alpha ; p, q, r)=\left\{\begin{array}{cc}
0, p & z \leq p \\
\frac{z-p}{q-p}, & p \leq z \leq q \\
\frac{r-z}{r-q}, & q \leq z \leq r \\
0, & r \leq z
\end{array}\right\}
$$

where $p$ denotes the minimum value of $z, r$ is the maximum value of $z$ and $q$ is the middle value which is determined by $p$ and $r$. Thus, the value $\alpha$ is evaluated by the aforementioned membership function. Then, the $\beta$ value is derived as

$$
\beta=1-\alpha .
$$

The values of $\alpha$ and $\beta$ are employed to design a new kernel function which is embedded into the spherical SVM classifier for better recognition performance. Figure 4 represents the triangular membership function.

4.3b Fuzzy spherical SVM-a new classifier: Once the kernel function is developed, it is then incorporated with the spherical SVM classifier. Thus, the spherical SVM classifier is derived by using the violating vectors of the feature, and its corresponding weights, the centre of the enclosed ball, radius of the sphere and the stopping criterion. The proposed fuzzy-based spherical SVM classifier is derived as follows.

The extracted feature using the HOG descriptor that is represented in the proposed classifier as $\left(j_{i}, k_{i}\right)$, where $i=1,2, \ldots, s, j_{i}$ is the feature vector, $k_{i}$ is the output class, and $s$ is the number of data samples. Then, the hyperplane function is defined by

$$
k_{i}\left(j_{i} \bullet w+h\right) \geq \lambda-\gamma_{i}
$$

with subject to $\left\|M-p_{i}\right\|^{2} \leq R^{2}$.

(i) Initialisation: During initialisation, the random feature vector is chosen and its weight is initialised to one. Then, the centre is estimated by the feature vectors along with its weights and radius is also deliberated [18] using the centre of the ball.

$$
\begin{gathered}
M=\sum_{i=1}^{s} \omega_{i} j_{i} \quad \text { where } \quad \omega \leftarrow 0, \omega_{0} \leftarrow 1 \\
R=\sqrt{\delta+1+\frac{1}{C}}, \quad \hat{\mu} \leftarrow \frac{1}{2}
\end{gathered}
$$

where $M$ is the centre, $\omega$ is the weight, $R$ is the radius and $\delta$ is constant.

(ii) Evaluate the violating vectors: For every iteration, the two violating vectors are generated by the number of draw attempts. At first, we have drawn the random subset 
of size $S_{r}$ from the whole dataset. Then, the first violating vector is estimated by the maximal distance between the feature vector and centre of the ball. After the violating vector is defined, the subset is drawn again by the number of draw attempts, $D_{a}$. Here, the second violating vector is declared by the minimal distance between the centre and feature. The violating vectors are generated by

$$
\begin{gathered}
V_{c} \leftarrow\left\|M-f_{i}\right\|>(1+\hat{\eta}) R \\
V_{d} \leftarrow\left\|M-f_{i}\right\|<(1+\hat{\eta}) R
\end{gathered}
$$

where $V_{c}$ and $V_{d}$ are the two violating vectors with respect to the centre of the sphere and feature vector.

(iii) Compute $\delta, \eta$ and $\hat{\eta}$ : By using the two violating vectors, we can upgrade the centre of the ball. This new centre is utilised to estimate the parameter $\hat{\eta}$. The new centre of the ball is calculated as

$$
M^{\prime}=M+\eta\left(V_{c}-V_{d}\right)
$$

Here, the new sphere of the centre $M^{\prime}$ touches the violating vector $V_{c}$, then, the radius should be satisfied by the following condition:

$$
\left\|M^{\prime}-V_{c}\right\|=R .
$$

Then, Eq. (20) can be written with respect to the violating vectors and the centre of the sphere as

$$
\left\|M+\eta\left(V_{c}-V_{d}\right)-V_{c}\right\|^{2}=R^{2} .
$$

Then, the parameter $\delta$ is computed by the two violating vectors and centre:

$$
\delta=\frac{\left(V_{c}-V_{d}\right) \times\left(V_{c}-M\right)}{K^{2}} .
$$

where $K$ is the newly developed multi-kernel function which constitutes exponential and tangential kernel function. Here, the kernel function is expressed by the dot products between the two violating vectors $V_{c}$ and $V_{d}$. The kernel function is the most important significant part in SVM classifier and can be used in many applications. The exponential and tangential kernel functions are used to implicit the feature space without a prior knowledge of the data coordinates in that space.

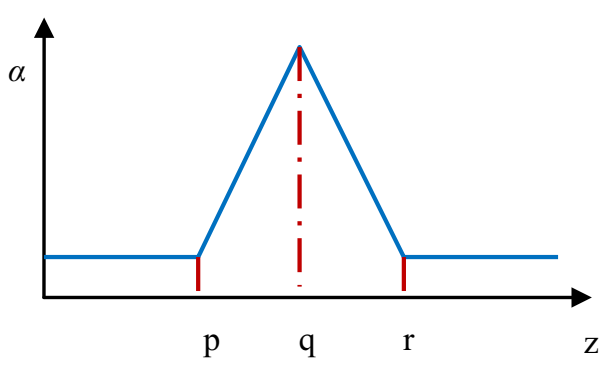

Figure 4. Triangular membership function.
Using $\eta$ and $R^{2}$ value, we can estimate the $\hat{\eta}$ by

$$
\hat{\eta}=\delta-\sqrt{\delta^{2}-\frac{K^{2}-R^{2}}{K^{2}}} .
$$

(iv) Updating the violating vector weights: The weights of the two violating vectors are denoted by $\omega_{c}$ and $\omega_{d}$. Thus, their weights are updated using the $\eta$ parameter, which is represented [18] by

$$
\eta=\min \left\{\hat{\eta}, V_{d}\right\} .
$$

Then, the weights are updated by

$$
\begin{aligned}
& \omega_{c} \leftarrow \omega_{c}+\eta \\
& \omega_{d} \leftarrow \omega_{d}-\eta
\end{aligned}
$$

where $\omega_{c}$ and $\omega_{d}$ denote the weights of two violating vectors $V_{c}$ and $V_{d}$ and $\eta$ is the parameter used to update the vector weights, which then leads to upgrading the centre of the sphere in the proposed classifier. Finally, in the proposed classifier, the updating process is repeated until the stopping criterion is reached.

\section{Results and discussion}

This section discusses the experimental results of our proposed system, and the performance is analysed by parameters such as FAR (false acceptance rate), FRR (False Rejection Rate) and accuracy. Then, the performance is compared with the existing systems.

\subsection{Experimental results}

The experimental results are calculated for the proposed fuzzy-based multi kernel spherical SVM classifier, which is implemented in MATLAB. Thus, the experimental results are apparently deliberated as follows.

5.1a Dataset description: The chars74K [19] database is employed in this paper to obtain the effective handwritten OCR. The required dataset is taken from this database which is given as input image to the proposed system. The chark74K dataset constitutes both the English and Kannada characters. Here, we utilised the English handwritten characters for our experimentation and performance analysis. Thus, the chars74K dataset consists of:

- 64 classes are composed of an upper case, lower case and numbers (A-Z, a-Z, 0-9).

- 7,705 characters are obtained from the natural images.

- 3,410 handwritten characters are drawn using tablet PC.

- 62,992 synthesised characters from computer fonts.

5.1b Evaluation parameters: The performance is analysed using FAR, FRR and accuracy based on the percentage of training data, number of draw attempts and stopping 
criterion. Then, the analysed performance is compared with existing systems such as hdes+lmnn (HOG descriptor+Levenberg-Marquardt Neural Network), gdes+lmnn (Grid descriptor+ Levenberg-Marquardt Neural Network), hybrid+lmnn, hdes+flmn (HOG descriptor+ Firefly Neural Network), gdes+flmn (Grid descriptor+ Firefly Neural Network), hybrid+flmn and hybrid classifier.

5.1c Classifiers taken for comparison: HDES+LMNN: This system is composed of both HOG descriptor [17] and Levenberg-Marquardt neural network [20]. The HOG descriptor is used to extract the features from the image. The histogram and normalisation are the two significant aspects in this descriptor. Then, the features are classified by the LMNN network. It is an algorithm which is employed to resolve the problem of minimising a function. It is fast and has stable convergence. Thus, the character image is recognised by the HOG and LMNN network.

GDES+LMNN: At first, the image is applied to the grid format, which provides the positional information precisely. Then, the feature vector is computed with respect to the grid level [21]. In grid-level feature, the binary vector is computed after mapping the input character image into the grid format based on the presence of the pixels. The LMNN network is utilised for character recognition. The Levenberg-Marquardt neural network [20] is a second-order approach to training the data without Hessian matrix. However, the efficiency of this network decreases when the number weight is increased.

Hybrid+LMNN: The LMNN with the hybrid system which comprises of HOG [17], grid-level feature [21] and GLCM (Grey Level Co-occurrence Matrix) [22]. The HOG descriptor extracts the features by applying the grid format to extract the local gradients and texture features using GLCM [22]. After the features are extracted, they are fed into the LMNN network [20] for classification.
HDES+FLMN: The HOG descriptor [17] and Firefly training algorithm [23] are used for character recognition. Here, the NN is trained based on the firefly algorithm to find the optimal weights, which are then used for classification. The HOG descriptor is employed to compute the feature elements and then it is fed into the firefly training algorithm for classification. The other existing system is composed of both grid-level feature [21] and firefly LM algorithm [23]. This system is also used for comparison with the proposed system, termed as GDES+FLMN.

Hybrid classifier: In this system, the features are extracted by the HOG feature [17], grid-level feature [21] using grid format and the grey-level co-occurrence matrix [22] is done by the matrix consisting of neighbouring and reference pixel values. Then, the hybrid classifier comprises Neural Network [20], Decision tree classifier [24] and a probabilistic model is employed for character recognition.

Fuzzy-based spherical SVM: Our proposed system contains the HOG descriptors [17] and spherical SVM classifier [18]. The histogram of the oriented gradient is used to extract the feature from the character image. Then, we develop a new kernel function based on the fuzzy triangular membership function, which is integrated with the spherical SVM classifier. Thus, the proposed classifier enhances the accuracy value when compared to the existing systems.

5.1d Experimental results: Figure 5 represents the experimental results of our proposed fuzzy-based spherical SVM classifier. Figure 5a shows the number of the character images as the input image. Then, the HOG feature descriptor is utilised to extract the feature by gradient calculation, gradient vote and normalisation computation. Thus, figure $5 \mathrm{~b}$ depicts the feature extracted image of the handwritten characters.

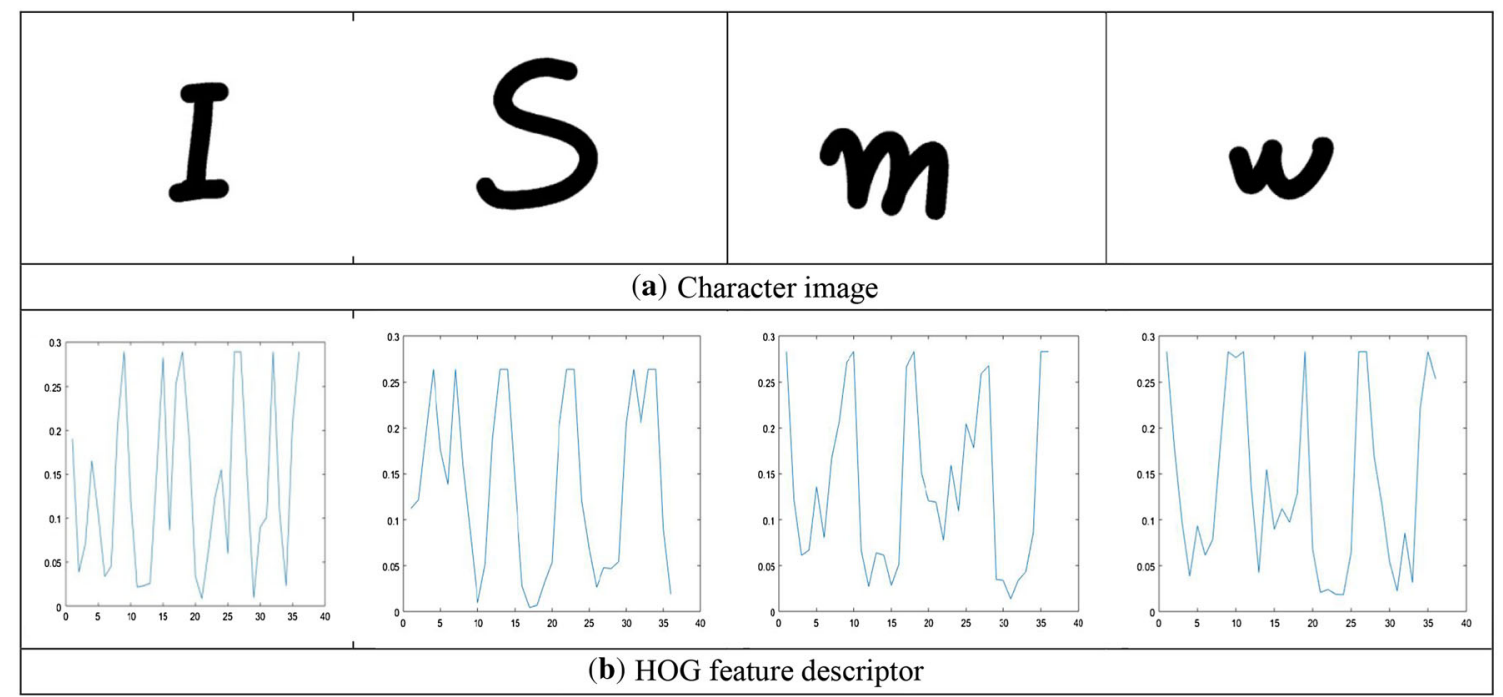

Figure 5. Experimental results. (a) Character image and (b) HOG feature descriptor. 


\subsection{Performance analysis}

Performance is analysed by metrics such as FAR, FRR and accuracy value. Here, effective handwritten character recognition is achieved by accuracy performance. Then, the analysis is compared with some existing systems based on the number of percentage of training data samples. Depending on the value of the centre of the sphere in the proposed classifier, the performance of FAR, FRR and accuracy are analysed.

5.2a Analysis using stopping criterion, $\varepsilon$ : The FAR, FRR and accuracy performance is analysed by varying the stopping criteria, $\varepsilon$, which is shown in figure 6 . Figure $6 \mathrm{a}$ depicts the FAR performance based on the centre of the sphere. The centre of the sphere is upgraded by the weights of violating vectors. The epsilon value provides the number of iterations in the proposed classifier, and it ranges from zero to one. The FAR is the false acceptance rate which is defined as the static measure of incorrectly recognised character by the correctly rejected characters. If the centre point is 0.25 , then the FAR achieved initially is 0.0968 . Then, by varying the epsilon value, the FAR is gradually decreased and increased, which is demonstrated in figure 6a. The false reject rate is determined by the ratio of incorrectly rejected characters and correctly recognised characters, which are expressed in percentage. Thus, the FRR performance is depicted in figure $6 \mathrm{~b}$. When the epsilon value is $0.0003,6.85 \%$ of FRR attained for centre point is $0.15,4.84 \%$ achieved for 0.20 centre point, and $8.06 \%$ FRR is obtained if the centre is 0.1. Comparatively, the FRR should contain the minimum value, which is acquired by the 0.25 centre value as shown in figure $6 \mathrm{~b}$. The accuracy is used to ensure the efficiency of character recognition system. Figure $6 \mathrm{c}$ represents the analysis of accuracy (a)

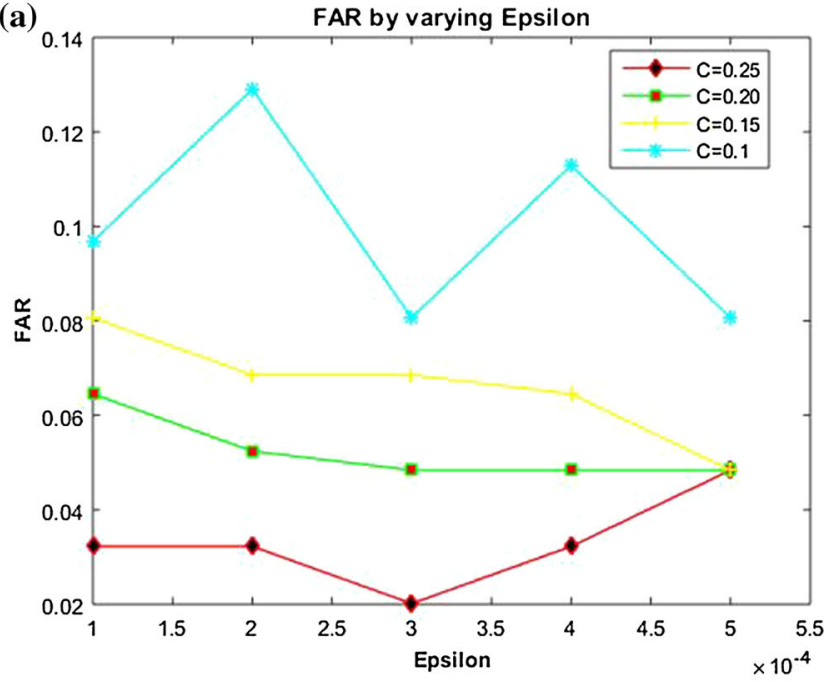

(b)

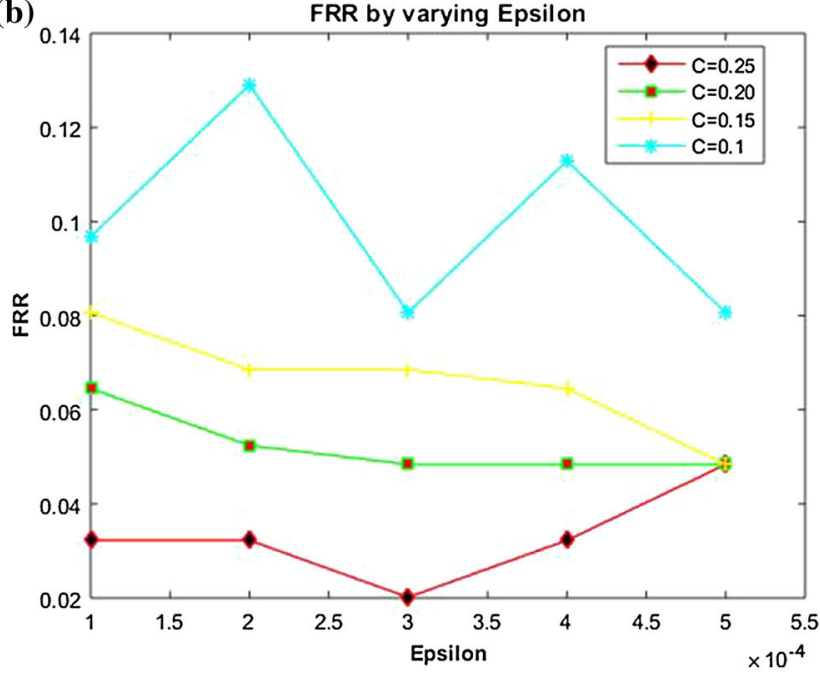

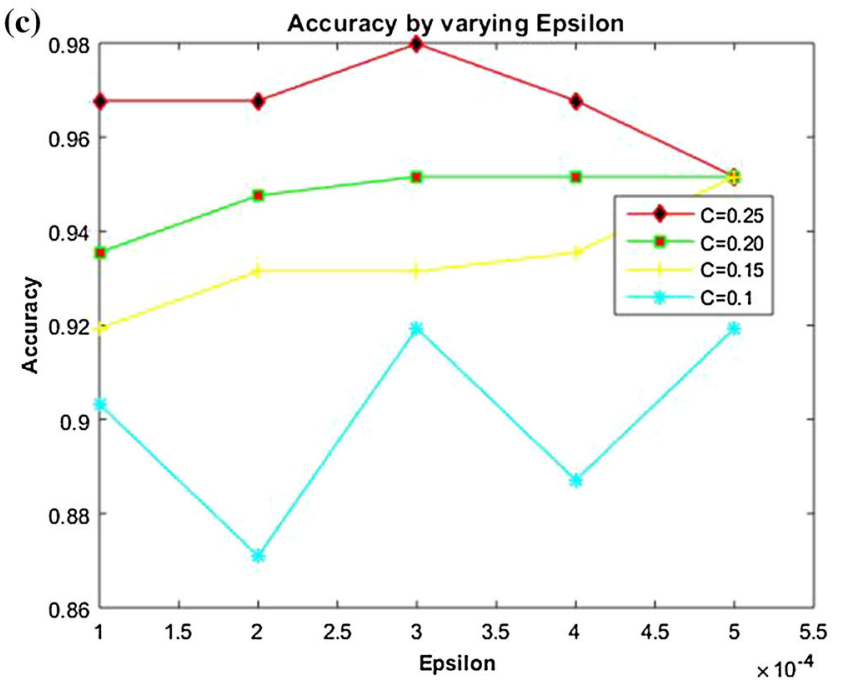

Figure 6. Performance analysis based on the epsilon (c). (a) FAR, (b) FRR and (c) accuracy. 
performance. If the centre point is $0.15,91.9 \%$ of accuracy is obtained when the stopping criteria are 0.0001 . Then, the accuracy is moderately increased by varying the epsilon value, which is shown in figure $6 c$.

5.2b Analysis by number of draw attempts, $D_{a}$ : Figure 7 demonstrates the performance analysis for the FAR, FRR and accuracy parameters. Based on the number of draw attempts, the evaluation metrics are analysed. The number of draw attempts (iteration) is used in the spherical SVM classifier to generate the violating vectors. The FAR rate of the proposed classifier is shown in figure $7 \mathrm{a}$. When the centre point is 0.25 , it sustains the $3.23 \%$ of FAR for all the number of draw attempts. Among the various centre values, 0.25 attains the very low false accept value, which is shown in figure $7 \mathrm{a}$. Figure $7 \mathrm{~b}$ depicts the FRR performance of our proposed system. When the number of draw attempts is 30 , the $9.27 \%$ of false reject is achieved at 0.1 centre of the sphere, the $8.06 \%$ value is attained for 0.15 centre value and acquires the $6.45 \%$ FRR, while the centre point is 0.20 . But figure $7 \mathrm{~b}$ demonstrates that the very low value of $3.23 \%$ false reject is attained at 0.25 centre point, which makes sure to enhance the accuracy value significantly. The accuracy performance is shown in figure $7 \mathrm{c}$. Due to the minimum value of FAR and FRR, the accuracy is improved efficiently for our proposed system. When the number of draw attempt is 20 , the accuracy value obtained depends on the centre point, which is demonstrated in figure $7 \mathrm{c}$. Accuracy of $88.71 \%, 92.74 \%, 93.55 \%$ and $96.77 \%$ is achieved at 0.1 , $0.15,0.20$ and 0.25 centre values in the proposed classifier. Thus, our proposed system provides better performance by attaining $96.7 \%$ accuracy, which is represented in figure $7 \mathrm{c}$.

5.2c Analysis by the percentage of training data: Depending on the percentage of training data samples, figure 8 depicted the accuracy performance and compared with an (a)

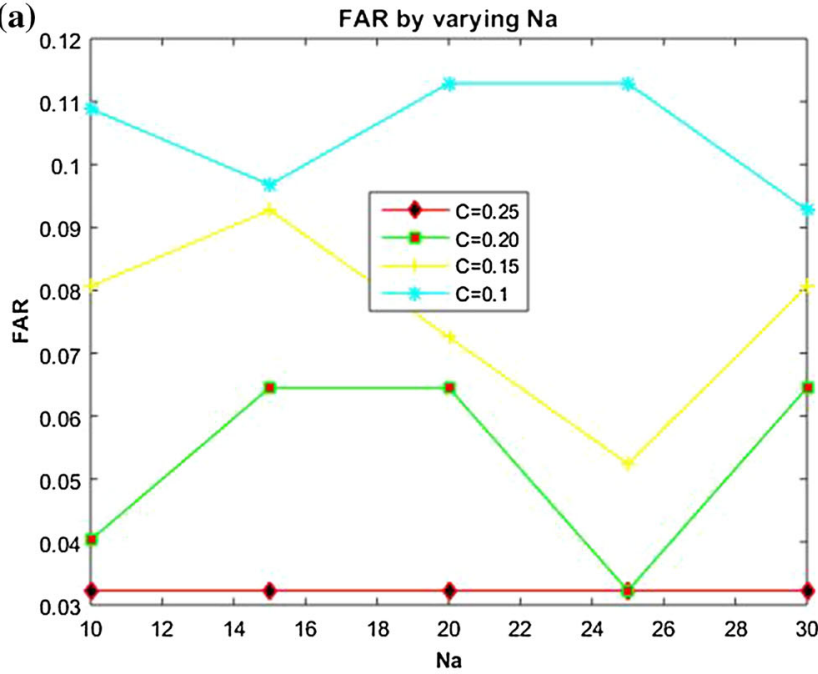

(b)

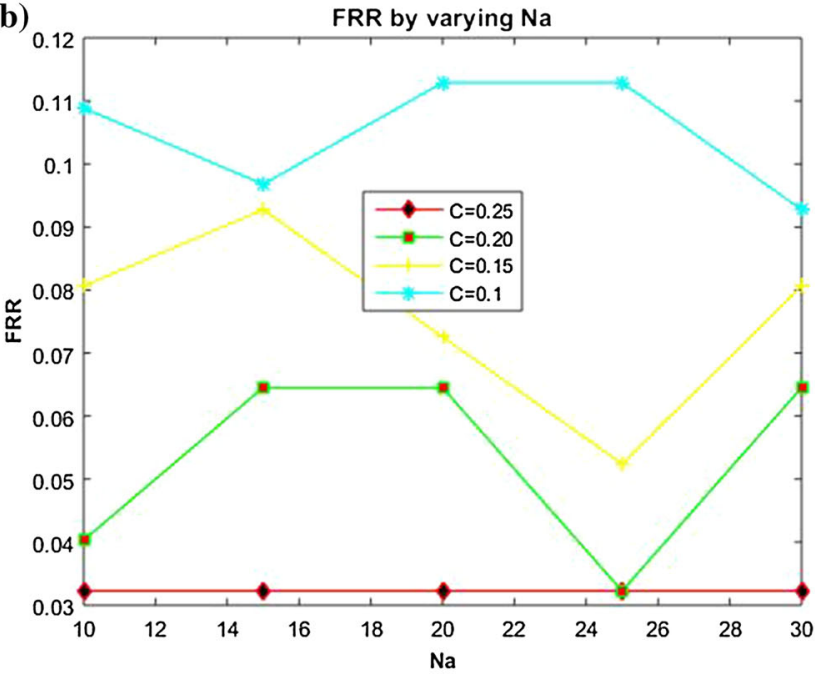

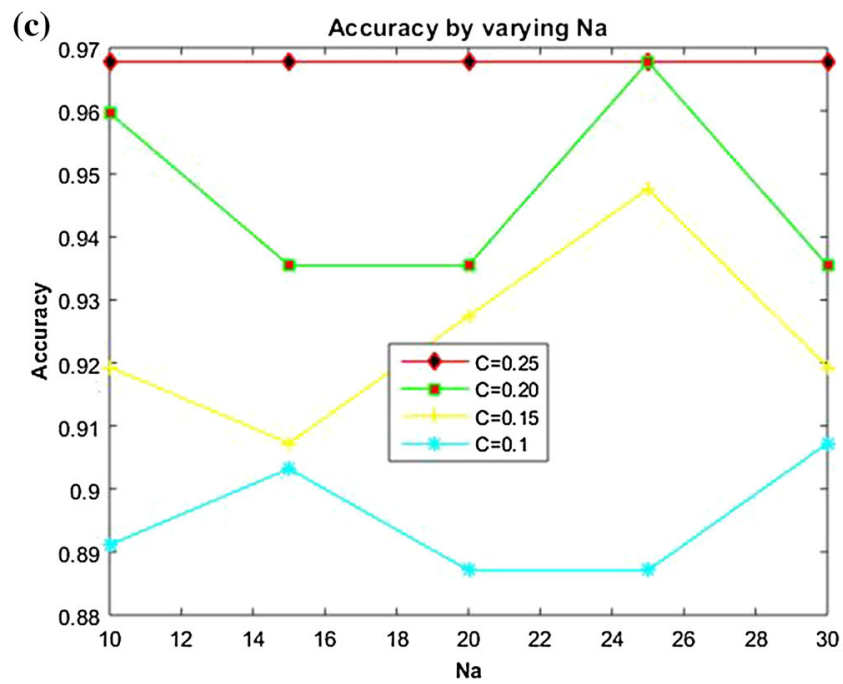

Figure 7. Performance analysis based on number of draw attempts. (a) FAR, (b) FRR and (c) accuracy. 
existing system such as hdes+lmnn, gdes+lmnn, hybrid+lmnn, gdes+flmn, hdes+flmn, hybrid+flmn and energy with hybrid classifier. The FAR is the measure of wrongly recognised character image with respect to the authorised character. Thus, figure 8a shows the FAR performance. When the classifier contains $70 \%$ of training data samples, the existing system like hdes+lmnn and hybrid classifier achieves $28 \%$ of FAR value. Then, the existing hdes+flmn and hybrid+lmnn methods attain $17 \%$ false accept rate and then $15 \%$ of FAR obtained in the system are by gdes+lmnn, gdes + flmn and hybrid + flmn methods. However, our proposed fuzzy sphere classifier acquires the very low value of 5.3\% FAR when compared to the existing systems, which is demonstrated in figure $8 \mathrm{a}$. The analysis of FRR performance is represented in figure $8 b$. The existing energy with hybrid classifier method achieves $28.5 \%$ FRR when the training data is 50\%. Then, the FRR value is gradually increased to $29.2 \%$ while increasing the percentage of training data samples, which is shown in figure $8 \mathrm{~b}$. However, our proposed classifier attains the minimum FRR value when compared with the existing systems. Figure $8 \mathrm{c}$ demonstrates the accuracy performance analysis. When the percentage of training data sample is 60 , the existing system acquires accuracy of $73 \%, 83 \%, 85 \%$ and $86 \%$. But figure $8 \mathrm{c}$ depicts that our proposed fuzzybased classifier achieves higher accuracy of $99.4 \%$ value when compared with hdes+lmnn, gdes+lmnn, hdes+flmn, gdes+flmn, hybrid+lmnn, hybrid+flmn and a hybrid classifier which provides better recognition performance.

\subsection{Comparative analysis}

Table 1 shows the comparative analysis of different methods on Chars74K data using accuracy. From this table, we clearly understand that the proposed method achieved the maximum accuracy of $99.4 \%$ accuracy in Chars $74 \mathrm{~K}$
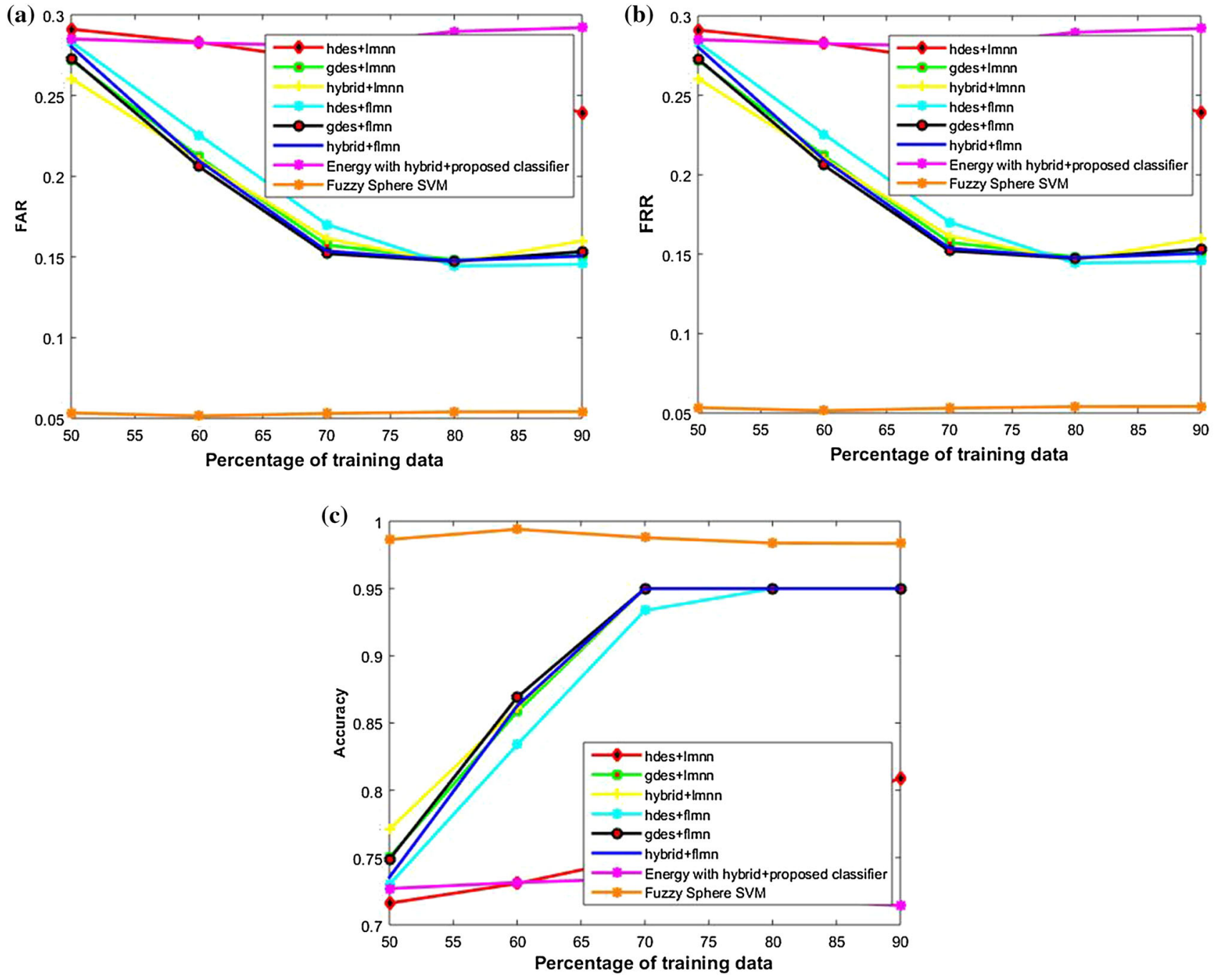

Figure 8. Performance analysis-based percentage of training data. (a) FAR, (b) FRR and (c) accuracy. 
Table 1. Comparative analysis of different methods on Chars74K data using accuracy.

\begin{tabular}{lc}
\hline Methods & Accuracy (\%) \\
\hline Proposed & 99.4 \\
Fuzzy spherical SVM & 73.44 \\
hdes+lmnn & 80.92 \\
gdes+lmnn & 95 \\
hybrid+lmnn & 95 \\
hdes+flmn & 95 \\
gdes+flmn & 95 \\
hybrid+flmn & 95 \\
Yash Sinha et al [25] & 73.16 \\
Fraz et al [26] & 72 \\
Shi et al [27] & 69.9 \\
Newell et al [28] & 66.5 \\
De Campos et al [29] & 55.26 \\
\hline
\end{tabular}

datasets. The root methods of the proposed methods, such as fuzzy spherical SVM, hdes+lmnn, gdes+lmnn, hybrid+lmnn, hdes+flmn, gdes+flmn and hybrid+flmn achieved the accuracy of $73.44 \%, 80.92 \%, 95 \%, 95 \%, 95 \%$, $95 \%$ and $95 \%$, respectively. While compared with these methods, the proposed methods obtained the maximum accuracy. The existing works, such as Sinha et al [25], Fraz et al [26], Shi et al [27], Newell et al [28] and Campos et al [29] obtained the accuracy of $73.16 \%, 72 \%, 69.9 \%, 66.5 \%$ and $55.26 \%$, respectively.

\section{Conclusion}

The fuzzy-based multi-kernel spherical SVM classifier was proposed in this paper for character recognition performance. The handwritten characters were varied from person to person by way of writing style, shape and rotation in both upper case and lower case. The proposed methodology was followed through the preprocessing, feature extraction and classification. Initially, the resizing operation was utilised in the preprocessing step to acquire the suitable image for further step. Then, the feature of character was extracted by the gradient histogram and normalisation of the image. These two methods were part of the HOG descriptor. Finally, the features were fed into the classifier to achieve the better recognition. Here, we proposed a fuzzy-based multi-kernel spherical SVM classifier for classification. The fuzzy triangular membership function was exploited in the proposed classifier to design a new multi-kernel function. Then, a novel multi-kernel function was integrated with the spherical SVM classifier to attain higher-accuracy performance. Thus, the experimental results of character image were determined, and the evaluation metrics analysed the performance. The outcome of our proposed system achieved the $99 \%$ of higher accuracy value, which provided the efficient handwritten character recognition.

\section{References}

[1] Kamble P M and Hegadi R S 2015 Handwritten Marathi character recognition using R-HOG feature. Procedia Comput. Sci. 45: 266-274

[2] Bhowmik T K, Ghanty P, Roy A and Parui S K 2008 SVMbased hierarchical architectures for handwritten Bangla character recognition. Int. J. Document Anal. Recognit. 12: 97-108

[3] Katiyar G and Mehfuz S 2016 A hybrid recognition system for off-line handwritten characters. SpringerPlus 5: 357

[4] Nasien D, Haron H and Siti Sophiayati Yuhaniz 2010 Support vector machine (SVM) for English handwritten character recognition. IEEE Computer Society

[5] Pradeep J, Srinivasan E and Himavathi S 2012 Performance analysis of hybrid feature extraction technique for recognizing English handwritten characters. IEEE

[6] Cheng-Lin Liu 2007 Normalization-cooperated gradient feature extraction for handwritten character recognition. IEEE Trans. Pattern Anal. Mach. Intell. 29(8): 1465-1469

[7] Sastry P N, Vijaya Lakshmi T R, Koteswara Rao N V and Abdul Wahab 2014 Telugu handwritten character recognition using zoning features. IEEE

[8] Prevost L, Oudot L and Moises A 2005 Hybrid generative/ discriminative classifier for unconstrained character recognition. Pattern Recognit. Lett. 26: 840-848

[9] Joseph S M, Abdu Rahiman V and Abdul Hameed K M 2015 SVM based feature set analysis in dynamic Malayalam handwritten character recognition. IEEE International Conference on Signal and Image Processing Applications (ICSIPA)

[10] Basu S, Das N, Sarkar R and Kundu M 2009 A hierarchical approach to recognition of handwritten Bangla characters. Pattern Recognit. 42: 1467-1484

[11] De Stefano C, Fontanella F, Marrocco C and Scotto di Freca A 2014 A GA-based feature selection approach with an application to handwritten character recognition. Pattern Recognit. Lett. 35: 130-141

[12] Surinta O, Karaaba M F, Lambert R B Schomaker and Wiering M A 2015 Recognition of handwritten characters using local gradient feature descriptors. Eng. Appl. Artif. Intell. 45: 405-414

[13] Shanthi N and Duraiswamy K 2010 A novel SVM-based handwritten Tamil character recognition system. Pattern Anal. Appl. 13: 173-180

[14] Patel M and Thakkar S P 2015 Handwritten character recognition in English: a survey. Int. J. Adv. Res. Comput. Commun. Eng. 4(2)

[15] Hanmandlu M, Murali Mohan K R and Chakraborty S 2003 Unconstrained handwritten character recognition based on fuzzy logic. Pattern Recognit. 36: 603-623

[16] Patel D K, Sam T and Singh M K 2013 Multiresolution technique to handwritten English character recognition using learning rule and Euclidean distance metric. IEEE

[17] Dalal N, Triggs B, Schmid C, Soatto S and Tomasi C 2005 Histograms of oriented gradients for human detection. In: Proceedings of International Conference on Computer Vision and Pattern Recognition, pp. 886-893

[18] Strack R, Kecman V, Strack B and Qi Li 2013 Sphere support vector machines for large classification tasks. Neurocomputing 59-67 
[19] T.deCampos, The Chars $74 K$ dataset: character recognition in natural images. Available at http://www.ee.surrey.ac.uk/ CVSSP/demos/chars74k/, Accessed November, 2015

[20] Hongwei Liu 2010 On the Levenberg-Marquardt training method for feed-forward neural networks. In: Proceeding of the Sixth International Conference on Natural Computation (ICNC)

[21] Muhammad Faisal Zafar, Dzulkifli Mohamad and Razib M Othman 2007 On-line handwritten character recognition: an implementation of counterpropagation neural net. Int. J. Comput. Electr. Automat. Control Inform. Eng. 1(10)

[22] Mohanaiah P, Sathyanarayana P and GuruKumar L 2013 Image texture feature extraction using GLCM approach. Int. J. Sci. Res. Publ. 3(5): 1-5

[23] Brajevic I and Tuba M 2013 Training feed-forward neural networks using firefly algorithm. In: Proceedings of the 12th International Conference on Artificial Intelligence, Knowledge Engineering and Data Bases (AIKED'13): pp. 156-161

[24] Cha Sung-Hyuk and Tappert Charles C 2009 A genetic algorithm for constructing compact binary decision trees. $J$. Pattern Recognit. Res. 4(1): 1-13
[25] Sinha Y, Jain P and Kasliwal N 2015 Comparative study of preprocessing and classification methods in character recognition of natural scene images. Machine Intelligence and Signal Processing, Advances in Intelligent Systems and Computing. vol. 390, pp. 119-129

[26] Fraz M, Sarfraz M and Edirisinghe E 2014 Exploiting colour information for better scene text recognition. In: Proceedings of the British Machine Vision Conference. BMVA Press

[27] Shi C Z, Wang C H, Xiao B H, Zhang Y and Gao S 2014 Multiscale graph-matching based kernel for character recognition from natural scenes. Acta Autom. Sin. 40: 751-756

[28] Newell A J and Griffin L D 2011 Natural image character recognition using oriented basic image features. In: Proceedings of IEEE International Conference on Digital Image Computing Techniques and Applications (DICTA), pp. 191-196

[29] De Campos T E, Babu B R and Varma M 2009 Character recognition in natural images. In: Proceedings of the International Conference on Computer Vision Theory and Applications, pp. 1-6 